\title{
Face Perception In Tetrastable Visual Awareness
}

\author{
Ahmad Yousef ${ }^{1}$ \\ 'School of Computational Science and Engineering, McMaster University, Hamilton, Ontario, Canada \\ *Correspondence: mohamas2@mcmaster.ca
}

\begin{abstract}
This article combines monocular rivalry with binocular rivalry in one setting. Astonishingly, this combination allows the human subjects to be visually aware about four different stable perceptions. Namely, they are aware of the two human faces, the vase, the duck, and the rabbit face in one setting. The visual awareness was stable with almost no mixed percepts. This experiment is a good measure of tetrastable visual perception for affective psychology and neuroscience, because it considers the perception of faces.

Introduction

Binocular rivalry, a phenomenon of visual perception in which human visual awareness alternates between two different visual stimuli presented to each eye, is an astonishing psychophysical approach to quantify consciousness, see reference 1 to 5 . Binocular rivalry is a form of bistable visual perception because it can stably alternate the human visual awareness. Monocular rivalry, however, is a phenomenon of the human perception that happens when
\end{abstract}

an ambiguous image is presented, for example duck-rabbit ambiguous image, see references 6 to 8 . The human visual awareness is therefore switching unstoppably between the duck face and the rabbit face; and thus monocular rivalry can be also considered as a form of

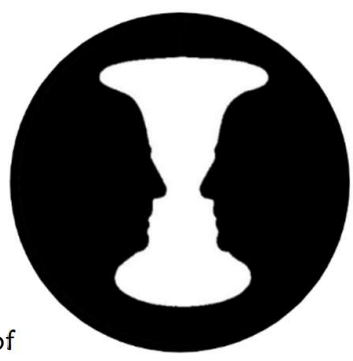
bistable visual perception, see reference 6 . Scientists believe that the human visual awareness might be able to interpret up to trinocular rivalry, namely, six stable rivalrous visual perceptions, see O'Shea et al. 2008; in the study, therefore, we had utilized two ambiguous images that cause monocular rivalry, namely, the duck-rabbit ambiguous image, and two faces - vase ambiguous image. Namely, we had built a binocular rivalry system, with stimuli that cause monocular rivalry, and thus, the production of a tetrastable visual perception. Initially, we had noticed that the duck face and rabbit face must appear in the visual awareness one after another, namely, the perception of the two faces or the vase, cannot appear between the perception of the duck, and the rabbit faces. The aforementioned observation is critical, namely, it concludes that monocular rivalry should be achieved before the binocular perceptual switch. We also noticed that the two human faces is the most dominate in accessing the visual awareness, but the vase is the most suppressed, it barely access the human visual awareness.
Materials \& Methods

Twelve human subjects, with dilated pupils, were recruited to participate in this study. To consolidate the conclusion, human subjects had participated in the entire experiment for two times; one in the morning and another one in afternoon. Each circle in each stimulus subtends exactly 8.76 DVA, namely, 3.7 centimeters, on the screen, whereas the human subjects are 24 centimeters away from the screen, with 9.25 diopters base-out glasses, see reference 9 . We had two trails for each of our twelve human subjects; the first trail uses the stimuli in the central images, and the second trail is a flipped one for counterbalancing purposes. For quantitative analysis, each trail lasted for five minutes, whereas a specialized timer had been recording every switch, key press 1 for two human faces, key press 2 for vase, key press 3 for duck, and key press 4 for rabbit, see reference 9 . After the behavioral data collection, the trails are averaged, and then standard error of the mean is estimated. In a sperate experiment, for further validation of our voluntary blinks' finding, we requested

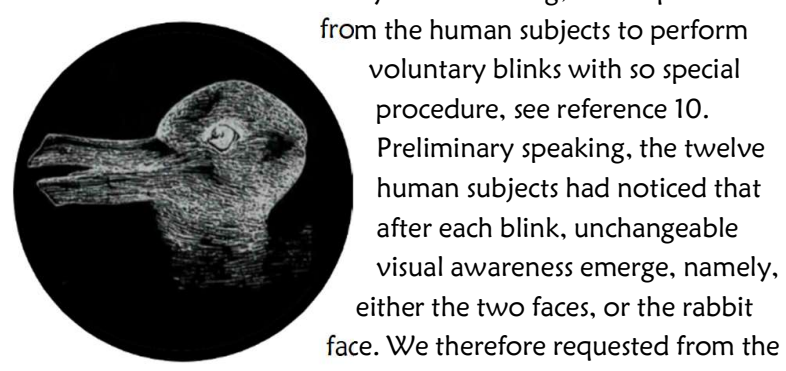
human subjects to verify whether they can keep their unchangeable visual awareness for two minutes, and all of them were able to achieve the aforementioned challenge. Most of the subjects reported seeing the duck face appear just after the rabbit face (monocular rivalry for one eye), but not the vase after the two faces. Since there is a phasic pupil dilation after each blink; these findings could be consistence with our previous study that theorized that the rabbit face is more dominant in dim lightening conditions (pupil dilation), see reference 11.

Results and Discussion

As shown in the central infographic, there is a clear tetrastable visual awareness, namely, the brain is_consciously awareness about four different entities despite the fact that the visual stimulus had been unchanged. It is noticed that the dominance duration of the two human faces is extremely high, moreover, the duck and the rabbit faces must appear one after another. Findings which tell us that ambiguous images may significantly elongate the dominance duration in 
binocular rivalry settings. Further elongation for dominance duration might be obtained if the stimulus are presented intermittently (Leopold et al., 2002). Interesting enough, we were able to validate our voluntary blinks' theory on this tetrastable stimulus too. In fact, all of the human subjects were able to see unchangeable visual awareness exclusively for two minutes. Importantly, we have previously theorized that the inhibitory neurons had been sending information to the consciousness, and the consciousness has spectrum, starting from oblivion and ending with metacognition, see reference, 13. Namely, we think that each distinct visual awareness produced by our tetrastable visual stimulus may have different neurological roads to the human visual awareness. In another word, the links that connect the retinal photoreceptors with their corresponding terminal neurological nodes 'the inhibitory neurons' which eventually might signal the consciousness should be distinct for each of the four different perceptual awareness. Importantly, one of these percepts, the vase but not the others, shall not be a product of singling the consciousness through the fusiform face area. Interesting to mention, the perception of vase has the shortest dominance duration but not the faces (two talking faces, rabbit face, \& duck face), this might because the oxygenated hemoglobin red blood cells might be greatly \& frequently visiting the fusiform face area over the other brain areas. Astonishingly, the differences of these dominance durations might be precisely interpreted under the umbrella of affective neuroscience, namely, the longest duration corresponds to two human faces 'talking to each other'; then the rabbit, an animal that has a face somehow closer to human; then the duck, an animated entity but with characteristics way far from humans' characteristics; and finally, the vase, an inanimate entity! This could be because we had used high contrast stimulus to promote pupil dilation, see Wang, etal. 2014; and therefore, human subjects might experience better face recognition, see reference 11 .

Essential Notifications

Stimuli and the BR setup can be found in reference 9. Readers who don't have binocular rivalry setup are extremely welcome to visit our lab. Due to the complexity of the stimuli, special BR glasses is a must to properly experience the effect; otherwise, interference problems may occur. We had not examined the effect of deep breathing in this study, but we expect to see further domination of the two faces, and possibly the rabbit face hypothetically because the fusiform face area and the primary olfactory cortex are neighbors, see reference 16 . Scholars with brain imaging and retinal imaging facilities are welcome to cooperate. Enquiries should be sent to the author. Important to mention, we had also noticed a very weird phenomena, namely, blue, and yellow masked lines in front of the two human faces, in addition to the duck, and the rabbit face, see reference 9. We think it's a product of blue-yellow opponent process which was highlighted in Hering's theory, however, given the fact that the stimulus color is white color which is a combination of yellow, and blue colors, we think that physics might a role in the aforementioned phenomenon. Deeper investigations might be provided in a further research.

Transactional References

[1] Levelt (1965). Binocular brightness averaging and contour information. British Journal of Psychology.

[2] Engle (1956). The role of content in binocular resolution. The American Journal of Psychology.

[3] Malek, etal. (2012). Binocular rivalry of spiral and linear moving random dot patterns in human observers.

[4] Fahle (1982). Binocular rivalry: suppression depends on orientation and spatial frequency. Vision Research.

[5] Malek, (2018). Generalizing Duchenne to sad expressions with binocular rivalry and perception ratings. Emotion.

[6] Weisstein, Eric W. "Rabbit-duck illusion". MathWorld.

[7] Yousef, A. 2019. "Two Distinct Fusiform Face Areas." PsyArXiv. doi:10.31234/osf.io/a8gzv.

[8] Robert O'Sheaa, Amanda Parkerb, David La Rooy, David Alaisb (2009). "Monocular rivalry exhibits three hallmarks of binocular rivalry: Evidence for common processes." Vision Research. [9]Stimuli and the BR system may be found here: https://drive.google.com/drive/folders/1xON1ZcrGOi_V1rLCibXApX5IHOkW2vX

[10] Yousef, A. 2019. "Voluntary Blinks Stop Binocular Rivalry." PsyArXiv. doi:10.31234/osf.io/z4jrn.

[11] Yousef, A. 2019. "Two Distinct Fusiform Face Areas." PsyArXiv. doi:10.31234/osf.io/a8gzv.

[12] D. Leopold, etal. (2002). Stable perception of visually ambiguous patterns. Nature Neuroscience.

[13] Yousef, A. 2019. "Consciousness Might Be Localized in Extra

Physical Dimensions." PsyArXiv. 2019.

[14] O'Shea et al. (2008). Trinocular rivalry? Perception, ECVP Abstract.

[15] Wang etal. (2014) Modulation of stimulus contrast on the human pupil orienting response, in E. Journal of Neuroscience.

[16] Yousef, A. 2020. "Deep Breathing Governs Binocular Rivalry." PsyArXiv. doi:10.31234/osf.io/zr3uk. 